\title{
La calidad en las bibliotecas universitarias biomédicas según sus usuarios
}

\section{Por Javier López-Gijón, Belén Ávila-Fernández, Ignacio-Javier Pérez-Gálvez y Enrique Herrera-Viedma}

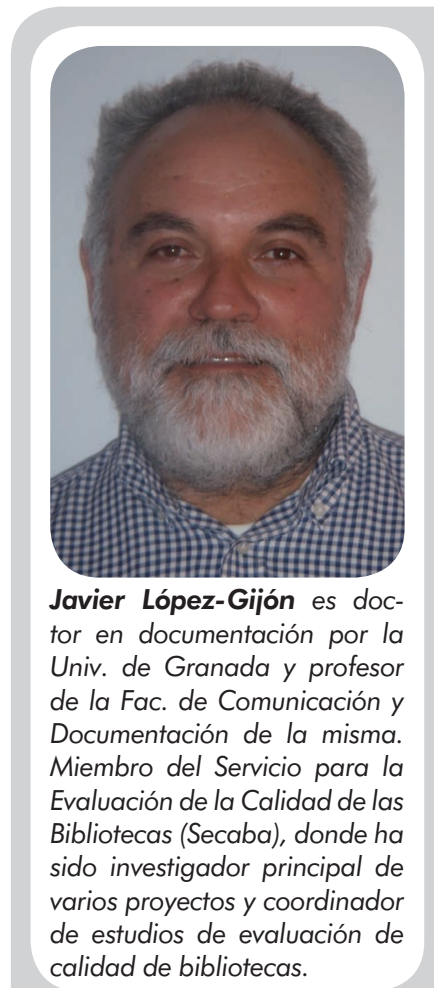

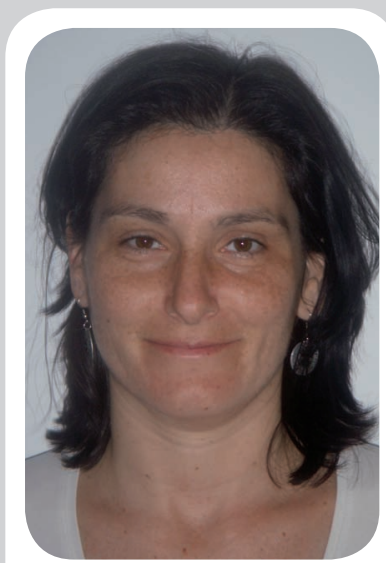

Belén Ávila-Fernández es licenciada en documentación por la Universidad de Granada. Actualmente es documentalista del Tribunal Superior de Justicia de Andalucía. Miembro del grupo Secaba, sus líneas de investigación se centran en la evaluación de la calidad en servicios de información y bibliotecas.

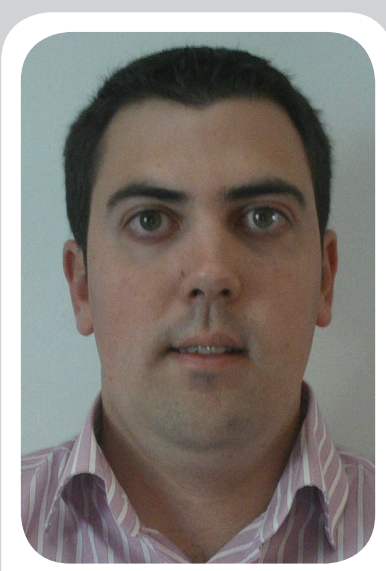

Ignacio-Javier Pérez-Gálvez es ingeniero informático por la Univ. de Granada y becario predoctoral en el Dpto. de Ciencias de la Computación e Inteligencia Artificial de la citada universidad. Miembro del grupo Secaba, investiga sobre toma de decisiones en ambiente difuso y evaluación de la calidad en bibliotecas.

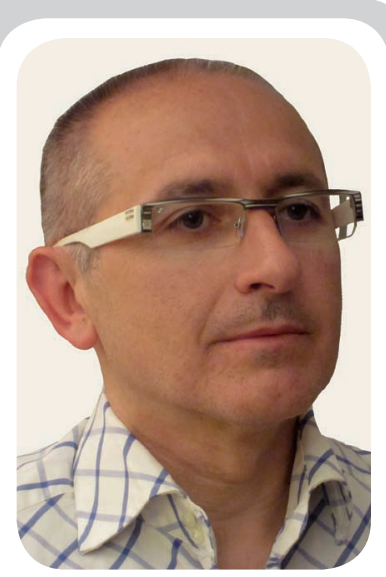

Enrique Herrera-Viedma es catedrático del Dpto. de Ciencias de la Computación e Inteligencia Artificial y Vicedecano de Investigación y Nuevas Tecnologías en la Facultad de Comunicación y Documentación de la Univ. de Granada. Es director del grupo Secaba y autor de más de 80 artículos en revistas JCR Presenta un índice $h=25$ según WoS.

Resumen: Se analiza la calidad de las bibliotecas universitarias biomédicas de España de acuerdo con lo que opinan, valoran y desean sus usuarios estudiantes. Sus opiniones se han recogido mediante la herramienta de evaluación de calidad de bibliotecas basada en satisfacción de usuarios LibQUAL+.

Palabras clave: Bibliotecas universitarias biomédicas, Satisfacción de usuarios, Calidad, LibQUAL+.

Title: The quality of biomedical academic libraries according to the users

Abstract: We analyze the quality of several academic heath libraries in Spain according to the users'opinions. We obtain these opinions using the tool to evaluate quality of libraries called LibQUAL+.

Keywords: Health academic libraries, User satisfaction, Quality, LibQUAL+.

López-Gijón, Javier; Ávila-Fernández, Belén; Pérez-Gálvez, Ignacio-Javier; Herrera-Viedma, Enrique. "La calidad en las bibliotecas universitarias biomédicas según sus usuarios”. El profesional de la información, 2010, mayo-junio, v. 19 , n. 3, pp. 255-259.

DOI: 10.3145/epi.2010.may.05

\section{Introducción}

La calidad es una filosofía considerada como punto central de los negocios o las actividades de una organización, involucrando a todas las funciones y a todos sus integrantes.

¿Cómo medimos la calidad en una biblioteca? Si seguimos el modelo planteado por Zeithaml, Parasuraman y Berry (1990) en el que "sólo los usuarios juzgan la calidad, los demás juicios son irrelevantes", diremos que una biblioteca será de calidad en la medida que responda a las necesidades de sus usuarios. Bajo esta perspectiva se deben utilizar modelos que permitan valorar la calidad de una biblioteca según las opiniones de los usuarios, como la metodología LibQUAL+.

Para Thompson, Kyrillidou y Cook (2007) este sistema "ayuda al personal de la biblioteca a entender las opiniones de los usuarios, y de esta manera mejorar la calidad de sus servicios y atender mejor sus necesida- 
des de información". Entre los objetivos -y como plantean diversos autores (Wilson; Town, 2006)- vemos que una de las grandes ventajas de la encuesta es que permite localizar las buenas prácticas y nos posibilita hacer benchmarking. Como opinan Játiva-Miralles y Gallo-León (2006), si en las bibliotecas universitarias españolas se impulsara una iniciativa para una valoración cualitativa de los servicios mediante el análisis de las percepciones que los usuarios tienen se "incrementarían de manera muy apreciable nuestras posibilidades de benchmarking". LibQUAL+ es considerado un estándar de evaluación de calidad de bibliotecas y ha sido utilizado en el análisis de un gran número de ellas, de distintos tipos y de diferentes países.

\section{"LibQUAL+ detecta la calidad de los servicios a partir de las percepciones de los usuarios"}

\section{Metodología}

Desde el grupo de investigación Servicio para la Evaluación de la Calidad de las Bibliotecas (Secaba), del que forman parte los autores de este trabajo, se está ayudando a muchas bibliotecas de la Red de Bibliotecas Universitarias españolas (Rebiun) a evaluar sus servicios usando LibQUAL+.

http://sci2s.ugr.es/secaba/

En este artículo se presentan los resultados más destacados obtenidos en la evaluación de calidad de una serie de bibliotecas universitarias biosanitarias mediante LibQUAL+ entre los años 2006 y 2009.

Las fechas en que se realizaron las capturas de datos en los centros son: Universidad $G$, Bibliotecas de Farmacia y Biosanitaria: febrero y marzo de 2006; Universidad $U$, Biblioteca de Enfermería: abril de 2007; Universidad M, Biblioteca de Medicina: abril de 2007; Universidad S, Biblioteca de Medicina y Enfermería: octubre y noviembre de 2009. Por cuestiones de compromiso de confidencialidad con los centros, no se pueden facilitar las denominaciones precisas de las universidades a las que pertenecen las bibliotecas estudiadas.

El método utilizado presenta una encuesta de 22 preguntas agrupadas en tres grandes dimensiones: $v a$ lor afectivo del servicio (VA); la biblioteca como lugar (Es) y el control de la información (CI). Esta última dimensión está relacionada con la colección y la información que suministra la biblioteca, hoy fuertemente determinadas por las herramientas y los medios electrónicos.

La encuesta tiene una escala de puntuación de 1 (el valor más bajo) a 9 (el máximo). En cada pregunta hay que responder tres cuestiones: nivel de servicio mínimo exigible (VM); nivel de servicio deseado (VD) y nivel observado (VO). El nivel mínimo que puntúa el usuario es aquel por debajo del cual piensa que el servicio no podría considerarse como tal. El nivel observado es el que el usuario percibe u observa en la biblioteca. Y el nivel deseado es la puntuación de cómo le gustaría que fuera el servicio.

La triple puntuación dificulta la recogida de datos pero permite conocer muy bien el grado de satisfacción con cada servicio. Sobre esa puntuación se definen dos variables, la adecuación del servicio (VO-VM) y la superioridad (VO-VD).

La adecuación nos indica si para el usuario el servicio que se presta es adecuado o no. Si el resultado es negativo quiere decir que no es adecuado, y por tanto los usuarios estarán insatisfechos. Por el contrario si tiene un valor positivo quiere decir que el servicio es adecuado para ellos. Cuanto mayor sea el valor obtenido más adecuado es.

La superioridad nos indica si el servicio que se presta es de calidad superior al deseado o no. Si el resultado es negativo quiere decir que no hay superioridad y por tanto tiene un margen de mejora. Cuanto mayor sea el valor obtenido, más superioridad tiene el servicio.

\section{Análisis de las bibliotecas universitarias biomédicas mediante LibQUAL+}

En la tabla 1 presentamos los resultados agregados obtenidos para las cinco bibliotecas biosanitarias estudiadas.

Algunas de las conclusiones que podemos extraer son las siguientes:

- Respondiendo a las preguntas, ¿qué desean los estudiantes? (valores deseados) y ¿en qué son más exigentes? (valores mínimos), podríamos concluir que lo que más desean son los espacios que les ofrece la biblioteca; y son más exigentes en el valor afectivo y en los espacios.

- Un usuario emergente se caracteriza por preferir el control de la información sobre las otras dimensiones (Lincoln, 2002). Según los resultados, este tipo de usuario no aparece todavía en estas bibliotecas, lo cual nos lleva a pensar que para ellos son prioritarias las acciones encaminadas a mejorar las otras dos dimensiones, o bien que la biblioteca necesitaría mejorar los recursos electrónicos y la formación en el uso de los mismos. 


\begin{tabular}{|c|c|c|c|c|c|}
\hline Estudiantes & $\begin{array}{l}\text { Farmacia } \\
\text { Uni. G }\end{array}$ & $\begin{array}{c}\text { Biosanitaria } \\
\text { Uni. G }\end{array}$ & $\begin{array}{c}\text { Enfermería } \\
\text { Uni. } \boldsymbol{U}\end{array}$ & $\begin{array}{l}\text { Medicina } \\
\text { Uni. M }\end{array}$ & $\begin{array}{c}\text { Medicina- } \\
\text { enfermería } \\
\text { Uni. S }\end{array}$ \\
\hline \multicolumn{6}{|c|}{ Valor afectivo del servicio } \\
\hline VM & 6,53 & 5,89 & 7,04 & 6,55 & 6,48 \\
\hline VO & 6,01 & 4,91 & 6,63 & 7,32 & 7,11 \\
\hline VD & 7,68 & 7,30 & 8,20 & 8,22 & 8,42 \\
\hline Adecuación & $-0,51$ & $-0,98$ & $-0,42$ & 0,77 & 0,63 \\
\hline Superioridad & $-1,66$ & $-2,39$ & $-1,57$ & $-0,90$ & $-1,31$ \\
\hline \multicolumn{6}{|c|}{ La biblioteca como espacio } \\
\hline VM & 6,23 & 6,51 & 6,46 & 6,69 & 6,43 \\
\hline VO & 5,71 & 5,94 & 5,78 & 6,87 & 6,50 \\
\hline VD & 7,47 & 7,74 & 8,37 & 8,33 & 8,29 \\
\hline Adecuación & $-0,53$ & $-0,57$ & $-0,68$ & 0,18 & 0,07 \\
\hline Superioridad & $-1,76$ & $-1,79$ & $-2,59$ & $-1,46$ & $-1,79$ \\
\hline \multicolumn{6}{|c|}{ Control de la información } \\
\hline VM & 6,09 & 5,64 & 6,11 & 6,48 & 6,68 \\
\hline VO & 5,28 & 5,08 & 5,84 & 7,37 & 6,87 \\
\hline VD & 6,73 & 6,90 & 8,16 & 8,02 & 8,30 \\
\hline Adecuación & $-0,80$ & $-0,57$ & $-0,27$ & 0,89 & 0,19 \\
\hline Superioridad & $-1,45$ & $-1,82$ & $-2,32$ & $-0,65$ & $-1,43$ \\
\hline
\end{tabular}

Tabla 1. $\square$ VM más altos, $\square$ VD más altos

- La superioridad es negativa en todos los casos y para todas las dimensiones, lo que nos hace pensar que hay un margen de mejora en cada una de ellas. De cara a decidir en qué dimensión actuar para mejorar, hemos de buscar en aquellas que no hay adecuación y actuar sobre las que se es más exigente. En el caso de la biblioteca Biosanitaria $G$, esto nos llevaría a recomendar actuar sobre la dimensión del espacio, aunque la adecuación sea mayor que en la dimensión afectiva.

\section{"LibQUAL+ presenta 22 preguntas agrupadas en tres dimensiones: valor afectivo del servicio, la biblioteca como lugar, y el control de la información"}

- La adecuación es negativa en todas las dimensiones en tres bibliotecas y positiva en todas las dimensiones en dos. Esto nos lleva a pensar que podría existir algún tipo de correlación entre las tres dimensiones consideradas en LibQUAL+: a) el VA muestra una alta correlación con el CI, de 0,891 (ver gráfico 1), b) el VA muestra también una alta correlación con el Es, de 0,936 (ver gráfico 2), c) el CI muestra también una alta correlación con el Es, de 0,872 (ver gráfico 3).

Algunas conclusiones que podemos extraer de estas correlaciones son:
- En sus orígenes $L i b Q U A L+$ contempló un mayor número de dimensiones, sin embargo finalmente se decidió sustentarlo sobre estas tres. Creemos que la alta correlación que encontramos justifica esa decisión y nos hace pensar que estas tres dimensiones constituyen el núcleo mínimo que hay que considerar para realizar la evaluación de calidad de una entidad que preste servicios a usuarios. En contextos bibliométricos cabría pensar que dado que correlacionan mucho, podríamos obviar el uso de dos de ellas y quedarnos tan sólo con una. Pero dado que valoran aspectos de distinta naturaleza creemos que la alta correlación refuerza el uso de las tres para evaluar la calidad de las bibliotecas, y nos hace pensar en la posibilidad de combinarlas en un único índice de evaluación para poderlo usar en rankings, como se hizo con los índices bibliométricos h y $\mathrm{g}$ en el trabajo de Herrera, Herrera-Viedma, Alonso y Cabrerizo (2009).

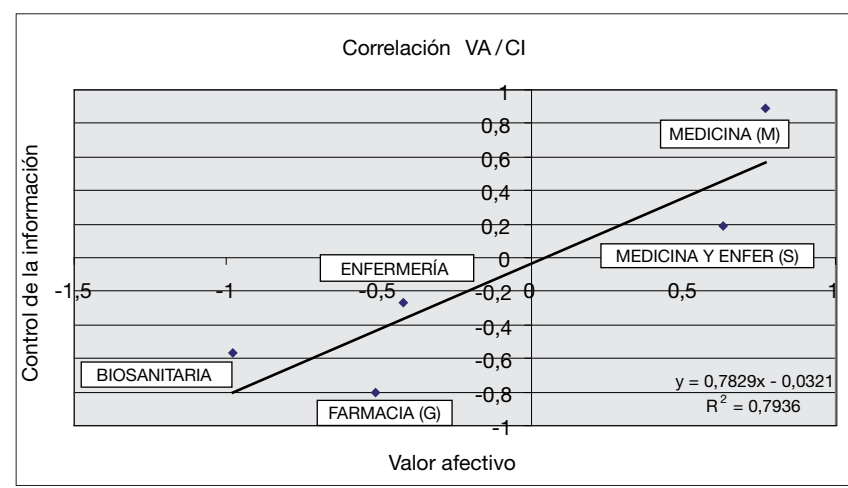

Gráfico 1. Correlación entre valor afectivo y control de la información 


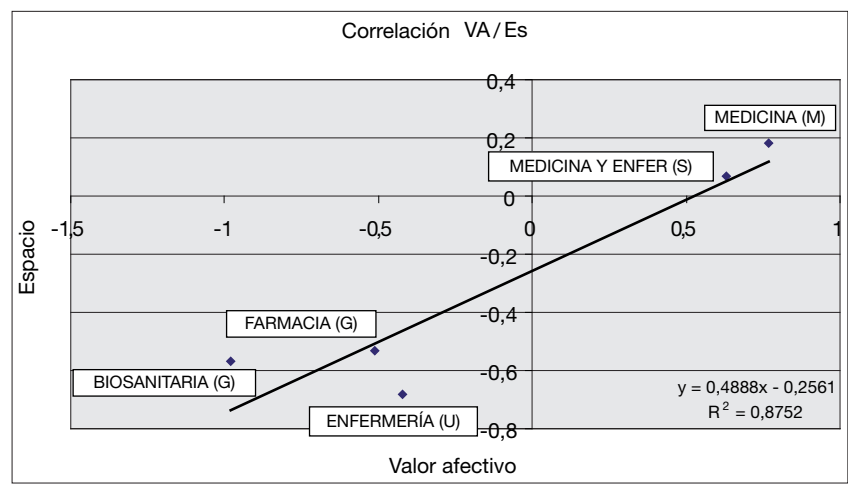

Gráfico 2. Correlación entre valor afectivo y espacio

- Que haya correlación entre estas variables quiere decir que están interrelacionadas y que la mejora de una llevará a la mejora de la otra, y viceversa. En el caso del CI y VA, esto significa que bibliotecas con VA adecuado (positivo) tenderán a tener el CI también positivo, y al contrario. Esta correlación nos lleva a pensar que el resultado que una biblioteca obtenga en el CI está determinado no sólo por las colecciones y materiales que oferte, sino también por el VA que los usuarios tengan con la biblioteca. Por tanto la percepción de la colección de una biblioteca está bastante determinada por el personal y podría suceder que dos bibliotecas con la misma colección pudieran tener resultados distintos en la dimensión CI si el VA de las mismas fuera en una positivo y en otra negativo (Herrera-Viedma; López-Gijón; Alonso; Vílchez-Pardo; Herranz-Navarra; Fernández-Porcel, 2007).

\section{"Los estudiantes de las bibliotecas universitarias biosanitarias demandan mejoras en los espacios y el valor afectivo"}

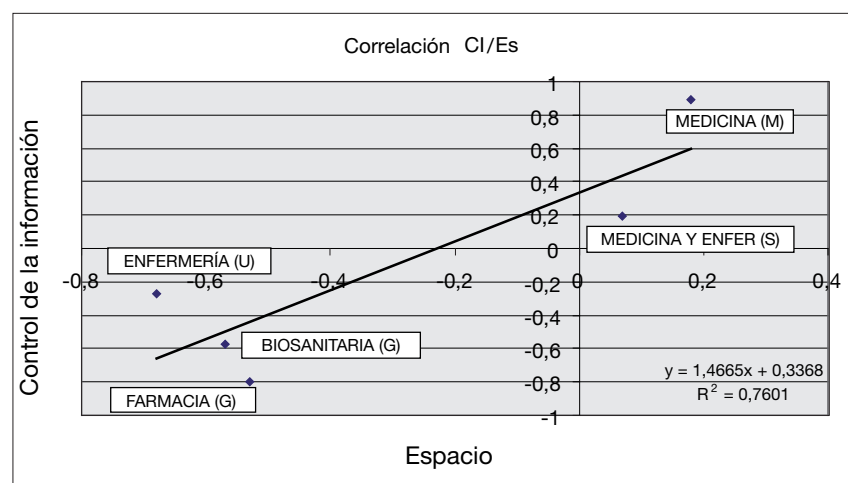

Gráfico 3. Correlación entre control de la información y espacio

Por tanto una colección se puede mejorar ofertando más documentos y otros aspectos relacionados con los servicios de información, pero también se puede mejorar la percepción que los usuarios tienen de la colección mejorando el VA. En el caso de la correlación entre el Es y CI, esto implica que cuando la biblioteca oferta mejores espacios (confort, silencio, diversidad de espacios, etc.) los usuarios tienen tendencia a pensar que la información que el centro les ofrece es mejor. Mejorando los espacios mejoramos no sólo la adecuación/satisfacción de los usuarios con los mismos, sino que podría afectar a la mejor adecuación del CI. En el caso de la correlación entre el VA y el Es, ésta nos indica que si mejorara la afectividad de los usuarios respecto al personal de la biblioteca, mejoraría también su percepción del espacio.

- Finalmente, si nos fijamos en los gráficos de correlación vemos que arriba y a la derecha aparecen las bibliotecas mejor valoradas por sus usuarios, las dos dimensiones que estamos analizando son adecuadas (positivas). Abajo y a la izquierda aparecen las bibliotecas poco valoradas por sus usuarios, las dos dimensiones son inadecuadas en estas bibliotecas. Esto nos permite identificar a las mejores bibliotecas en esas dimensiones y realizar procesos

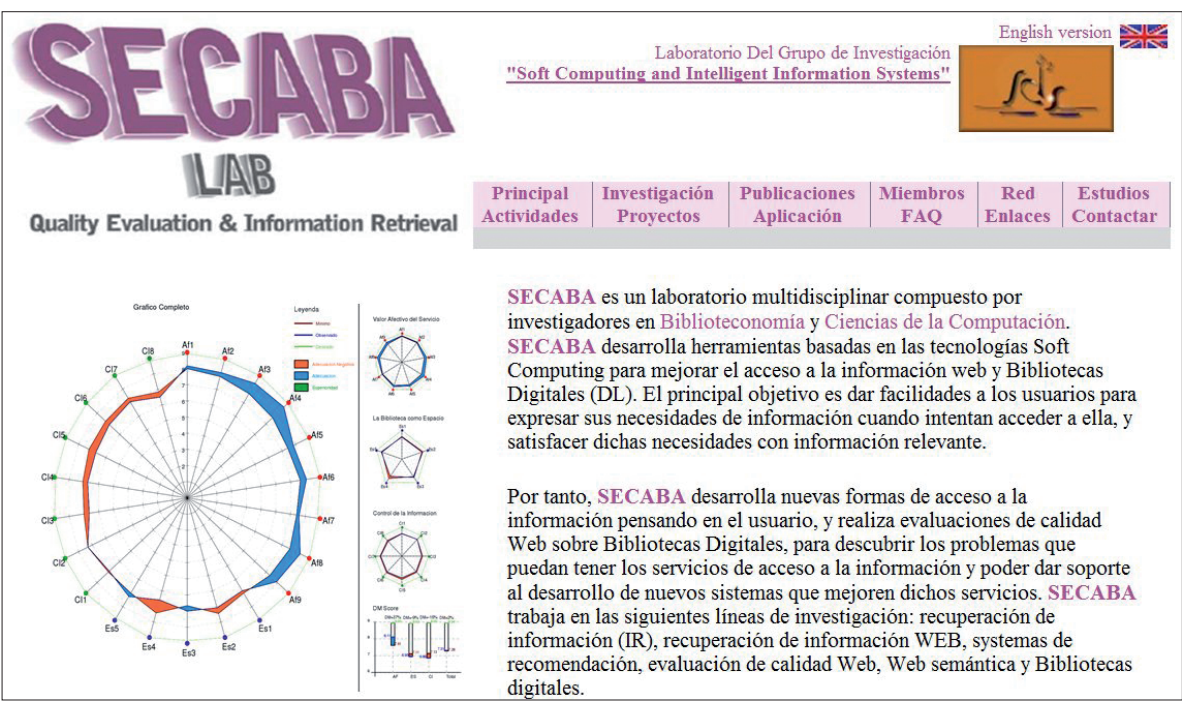

Servicio para la Evaluación de la Calidad de las Bibliotecas (Secaba), Universidad de Granada http://sci2s.ugr.es/secaba/ de benchmarking para guiar las políticas de mejora de aquellas que tengan carencias en ellas.

\section{Comentarios finales}

Teniendo en cuenta los resultados obtenidos podemos afirmar que los estudiantes de las bibliotecas universitarias biosanitarias demandan mejoras en los espacios y el valor afectivo, mientras muestran poco interés por el control de la información (las colecciones ofertadas). Además como una consecuencia de las altas correlaciones obser- 
vadas entre las dimensiones podemos decir que una biblioteca es un sistema en el que todos los elementos están relacionados y se afectan y por tanto, en una biblioteca todo es importante y todo afecta a todo.

\section{Referencias}

Herrera, Francisco; Herrera-Viedma, Enrique; Alonso, Sergio; Cabrerizo, Francisco-Javier. "Agregación de índices bibliométricos para evaluar la producción científica de los investigadores". El profesional de la información, 2009, v. 18, n.5, pp. 559-561.

Herrera-Viedma, Enrique; López-Gijón, Javier; Alonso, Sergio; Vílchez-Pardo, Josefina; Herranz-Navarra, Francisco; FernándezPorcel, Antonio. "Midiendo la satisfacción de usuarios para mejorar la calidad en las bibliotecas universitarias. En: 10as Jornadas españolas de documentación (Fesabid), 2007, pp. 317-325.

http://sci2s.ugr.es/publications/ficheros/fesabid2007.pdf

Játiva-Miralles, María-Victoria; Gallo-León, José-Pablo. "LibQual+ evaluación de calidad de servicios desde la perspectiva del usuario". Boletín de la Anabad, 2006, v. 56, n. 1, pp. 53-74.

http://www.anabad.org/organizacion/murcia/ATT00026.pdf

LibQUAL+. Charting library service quality.

http://www.libqual.org/about/about_lq/general_info
Lincoln, Yvonna S. "Insights into library services and users from qualitative research". Library and information science research, 2002, v. 24, n. 1, pp. 3-16.

Thompson, Bruce; Kyrillidou, Martha; Cook, Colleen. "User library service expectations in health science vs. other settings: a LibQUAL+ study". Health information and libraries journal, 2007, v. 24, n. s1, pp. 38-45.

Wilson, Frankie; Town, J. Stephen. "Benchmarking and library quality maturity". Performance measurement and metrics, 2006, v. 7, n. 2, pp. 7582.

http://www.emeraldinsight.com/Insight/viewContentItem.do? contentType= Article \& contentId $=1563577$

Zeithaml, Valeri A.; Parasuraman, A.; Berry, Leonard L. "Delivering quality service: balancing customer perceptions and expectations". New York: Free Press, 1990.

Javier López-Gijón, Belén Ávila-Fernández, IgnacioJavier Pérez-Gálvez, Enrique Herrera-Viedma. Universidad de Granada.

jgijon@ugr.es

bavila@ugr.es

ijperez@decsai.ugr.es

viedma@decsai.ugr.es

\section{Máster oficial \\ en Gestión de Contenidos Digitales \\ http://www.ub.edu/biblio/mged.html}
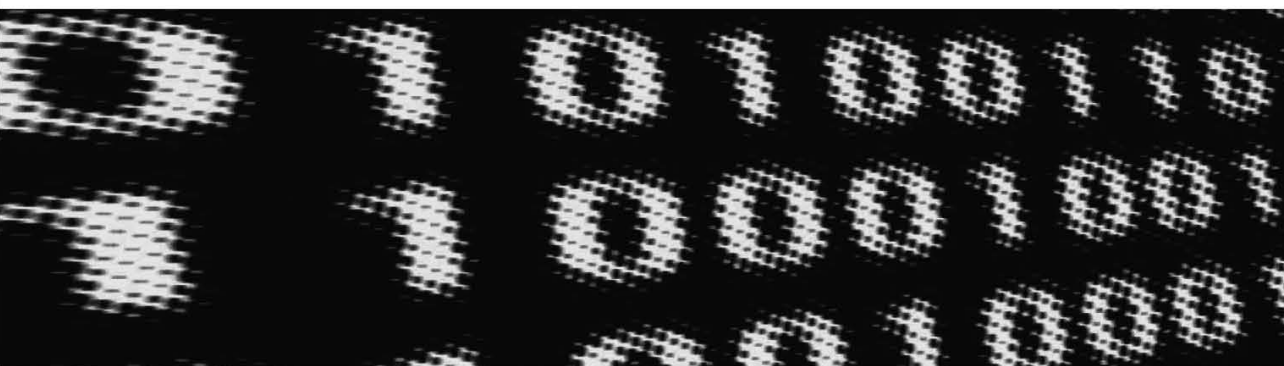

\section{CARACTERÍSTICAS}

Máster oficial e interuniversitario organizado desde el

curso 2005-2006

90 créditos ( 3 semestres)

Según los estudios cursados con anterioridad, se ha de seguir un módulo previo de nivelación

En horario de tarde, de 17 a $21 \mathrm{~h}$.

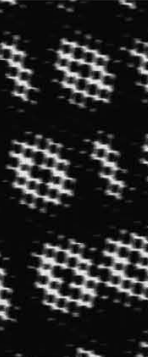

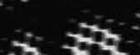

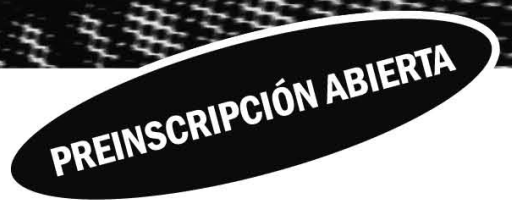

Más información

Universitat de Barcelona

Facultad de Biblioteconomía y Documentación

Melcior de Palau, 140 - 08014 Barcelona - Tel. 934035770

coord-mgcd@ub.edu 